\title{
Spectral self-interference fluorescence microscopy
}

L. Moiseev and C. R. CantorM. I. AksunM. Dogan and B. B. GoldbergA. K. Swan and M. S. Ünlü

Citation: Journal of Applied Physics 96, 5311 (2004); doi: 10.1063/1.1786665

View online: http://dx.doi.org/10.1063/1.1786665

View Table of Contents: http://aip.scitation.org/toc/jap/96/9

Published by the American Institute of Physics

\section{$A|P|$ Journal of Applied Physics}

Save your money for your research. It's now FREE to publish with us no page, color or publication charges apply.

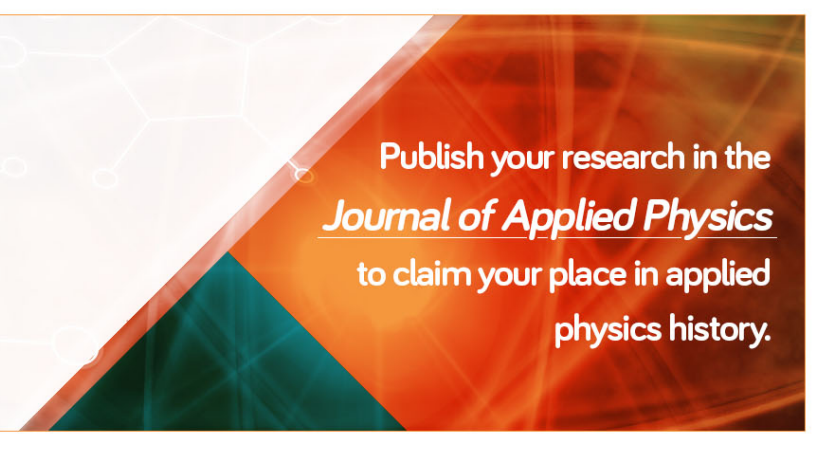




\title{
Spectral self-interference fluorescence microscopy
}

\author{
L. Moiseev and C. R. Cantor \\ Center for Advanced Biotechnology, 36 Cummington St. 2nd Floor, Boston, Massachusetts 02215
}

M. I. Aksun

Department of Electrical and Electronics Engineering, Koç University, Istanbul, Turkey

M. Dogan ${ }^{\text {a) }}$ and B. B. Goldberg

Department of Physics, Boston University, 590 Commonwealth Ave., Boston, Massachusetts 02215

\begin{abstract}
A. K. Swan and M. S. Ünlü
Department of Electrical and Computer Engineering, Boston University, 8 Saint Mary's Street, Boston, Massachusetts 02215
\end{abstract}

(Received 18 March 2004; accepted 30 June 2004)

\begin{abstract}
Spontaneous emission of fluorophores located close to a reflecting surface is modified by the interference between direct and reflected waves. The spectral patterns of fluorescent emission near reflecting surfaces can be precisely described with a classical model that considers the relative intensity and polarization state of direct and reflected waves depending on dipole orientation. An algorithm based on the emission model and polynomial fitting built into a software application can be used for fast and efficient analysis of self-interference spectra, yielding information about the location of the emitters with subnanometer precision. Spectral information was used to study thin films of fluorescent substances on surfaces. () 2004 American Institute of Physics.
\end{abstract}

[DOI: $10.1063 / 1.1786665]$

\section{INTRODUCTION}

It has been known for over a century that selfinterference of light creates standing waves above a reflecting surface. ${ }^{1}$ In a series of elegant experiments performed in the 1960s, the fluorescence decay of an organic dye embedded into a lipid layer was demonstrated to be an oscillatory function of its distance above a silver mirror. ${ }^{2}$ This observation led to an idea that the position of a dye above a mirror can be determined from the amount of fluorescent light it emits. ${ }^{3}$ In a recent related work, self-interference of light emitted by a single atom was observed to oscillate as a function of the distance between an atom and a mirror. ${ }^{4}$ A different technique, spectral self-interference fluorescence microscopy (SFM), also determines the height of fluorescent molecules above the surface, but via oscillations in the spectral, not spatial domain. ${ }^{5}$ When a light emitting dye is located at a distance of $(10-20) \lambda$ from a mirror, interference between the direct and the reflected waves produces several fringes in the emission spectrum. Figure 1(a) illustrates the effect of self-interference on the emission spectrum of fluorescein immobilized at different heights on a silicon-silicon oxide wafer [Fig. 1(b)]. This is compared with the smooth emission envelope of fluorescein immobilized on a glass slide where there is no self-interference.

In this paper we present an electromagnetic model of spectral self-interference to determine the precise position of fluorescent molecules above reflecting dielectric interfaces. The method is based on a classical model of dipole emission that predicts the shape of the oscillatory component of an emission spectrum modified by self-interference. This model

${ }^{a)}$ Electronic mail: mdogan@bu.edu is different from the previously published simulations of fluorescent emission near a mirror ${ }^{2,3}$ in that, it recognizes the relative intensity and polarization of light emitted in different directions by the dipole. We start with a general method for calculating the fields in the near field and far field. Then, only far field calculations are considered for the spectral selfinterference microscopy as the observation points, defined at the microscope objective, are always far from the source.

a)

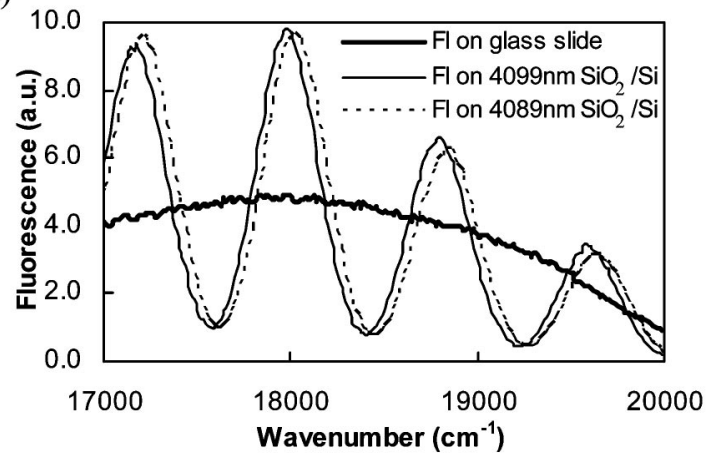

b)

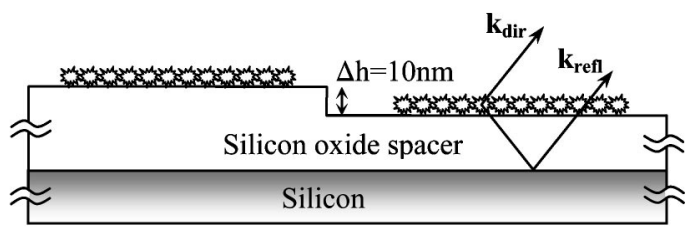

FIG. 1. (a) Emission spectra of fluorescein immobilized on a glass slide (free dipole) and on top of a $\mathrm{Si}_{-} \mathrm{SiO}_{2}$ chip with two different thicknesses of the oxide layer (10 nm difference). (b) Schematic of the $\mathrm{Si}_{-} \mathrm{SiO}_{2}$ chip used in the experiment (not to scale). 


\section{THEORY}

The problem can be defined as finding the electric and magnetic fields due to a fluorescent molecule modeled as an electric dipole in a multilayer planar medium. Once these are obtained, light emission intensity from a dipole, as collected by the objective, can be calculated by the integral of the Poynting vector over the surface of the objective. Therefore, the key step in this approach is to find the electric and magnetic fields at the plane of objective due to an arbitrarily oriented dipole in this geometrical setting. Note that the fields due to a dipole are usually referred to as Green's functions in electromagnetism (EM) community, and that the Green's functions in layered media are often obtained from their $k$-space representations, which can be obtained analytically in closed forms. ${ }^{6}$ Note that $k$-space $\left(\mathbf{k}=k_{x} \hat{x}+k_{y} \hat{y}+k_{z} \hat{z}\right.$, propagation constant) is usually referred to as spectral domain in the EM community, but since the term "spectral domain" is used for "frequency domain" in this work and previous related work, " $k$-space" is used throughout this work to refer to spectral domain in EM literature. For the $k$-space representation, we start with a plane wave expansion of a spherical wave originated from a point source in free space, and then the effects of the layered structure are taken into account by incorporating the reflections from the interfaces. Since there are mainly two types of reflection coefficients depending upon the polarization of the incident electric field, the plane wave constituents are grouped accordingly as TE and TM waves ( $s$ - and $p$-polarized waves) with the corresponding reflection coefficients $R_{\mathrm{TE}}$ and $R_{\mathrm{TM}}$. For example, the $k$-space representation of the electric field Green's function can be obtained for a multilayer planar geometry with a dipole located in the uppermost half space as follows.

(i) The primary fields due to a dipole in free space are expressed as if there was no boundary, as

$$
\tilde{\overline{\mathbf{G}}}_{0}^{\mathrm{E}}=\frac{\mu_{0}}{j 2}\left[\overline{\mathbf{F}}^{\mathrm{TM}}+\overline{\mathbf{F}}^{\mathrm{TE}}\right] e^{-j k_{z 0} 0} z^{-z_{0}} \mid,
$$

where the plane wave components of the spherical wave have been written in terms of TM and TE polarized plane waves, $\overline{\mathbf{F}}^{\mathrm{TM}}$ and $\overline{\mathbf{F}}^{\mathrm{TE}}$, respectively. Tilde $(\sim)$ over the Green's functions denotes the spectral-domain representation, bold letters denote vector quantities, while a bar over a bold letter denotes a dyad, $z_{0}$ is the location of the dipole over the topmost interface, and $k_{z 0}$ is the propagation constant of the plane waves in $z$ direction in the top layer (free-space in this example).

(ii) The Green's function for the reflected part of the electric field is expressed as

$$
\tilde{\overline{\mathbf{G}}}_{\mathrm{ref}}^{\mathrm{E}}=\frac{\mu_{0}}{j 2}\left[-R_{\mathrm{TM}} \overline{\mathbf{F}}_{\mathrm{ref}}^{\mathrm{TM}}+R_{\mathrm{TE}} \overline{\mathbf{F}}^{\mathrm{TE}}\right] e^{-j k_{z 0}\left|z-z_{0}\right|},
$$

where $R_{\mathrm{TM}}$ and $R_{\mathrm{TE}}$ take into account all possible multiple reflections from the layers below the interface, and hence are referred to as the generalized reflection coefficients for TM and TE polarized waves, respectively, at the topmost interface. ${ }^{7}$ Note that the generalized reflection coefficients reduce down to Fresnel's reflection coefficients in the case of a single semi-infinite layer below the interface.

(iii) Finally, the total electric field in the spatial domain is found as

$$
\mathbf{E}(\mathbf{r})=-j \omega\left[\overline{\mathbf{G}}_{0}^{\mathrm{E}}+\overline{\mathbf{G}}_{\mathrm{ref}}^{\mathrm{E}}\right] * \mathbf{J}(\mathbf{r}),
$$

where the spatial-domain Green's functions are obtained from the inverse Fourier transforms of their $k$-space representations. The operator $(*)$ denotes the dot product and the superposition integral. The current density $\mathbf{J}(\mathbf{r})$ of the point source is expressed as $\mathbf{J}(\mathbf{r})=\hat{\mu} I l \delta\left(\mathbf{r}-\mathbf{r}_{\mathbf{0}}\right)$ where $\hat{\mu}$ is the unit vector denoting the polarization of the dipole, $I l$ is the dipole moment, and $\mathbf{r}_{\mathbf{0}}$ is the location of the source. For the sake of completeness, let us give the TM and TE wave components of the primary fields and reflected fields. ${ }^{8}$

$$
\begin{aligned}
& \overline{\mathbf{F}}^{\mathrm{TM}}=\frac{1}{k_{0}^{2}\left(k_{x}^{2}+k_{y}^{2}\right)}\left[\begin{array}{ccc}
k_{x}^{2} k_{z 0} & k_{x} k_{y} k_{z 0} & \mp k_{x}\left(k_{x}^{2}+k_{y}^{2}\right) \\
k_{x} k_{y} k_{z 0} & k_{y}^{2} k_{z 0} & \mp k_{y}\left(k_{x}^{2}+k_{y}^{2}\right) \\
\mp k_{x}\left(k_{x}^{2}+k_{y}^{2}\right) & \mp k_{y}\left(k_{x}^{2}+k_{y}^{2}\right) & \left(k_{x}^{2}+k_{y}^{2}\right)^{2} / k_{z 0}
\end{array}\right],\left\{\begin{array}{l}
- \text { for } z>z_{0} \\
+ \text { for } z<z_{0},
\end{array}\right. \\
& \overline{\mathbf{F}}^{\mathrm{TE}}=\frac{1}{k_{z 0}\left(k_{x}^{2}+k_{y}^{2}\right)}\left[\begin{array}{ccc}
k_{y}^{2} & -k_{x} k_{y} & 0 \\
-k_{x} k_{y} & k_{x}^{2} & 0 \\
0 & 0 & 0
\end{array}\right], \\
& \overline{\mathbf{F}}_{\mathrm{ref}}^{\mathrm{TM}}=\frac{1}{k_{0}^{2}\left(k_{x}^{2}+k_{y}^{2}\right)}\left[\begin{array}{ccc}
k_{x}^{2} k_{z 0} & k_{x} k_{y} k_{z 0} & k_{x}\left(k_{x}^{2}+k_{y}^{2}\right) \\
k_{x} k_{y} k_{z 0} & k_{y}^{2} k_{z 0} & k_{y}\left(k_{x}^{2}+k_{y}^{2}\right) \\
-k_{x}\left(k_{x}^{2}+k_{y}^{2}\right) & -k_{y}\left(k_{x}^{2}+k_{y}^{2}\right) & -\left(k_{x}^{2}+k_{y}^{2}\right)^{2} / k_{z 0}
\end{array}\right],
\end{aligned}
$$




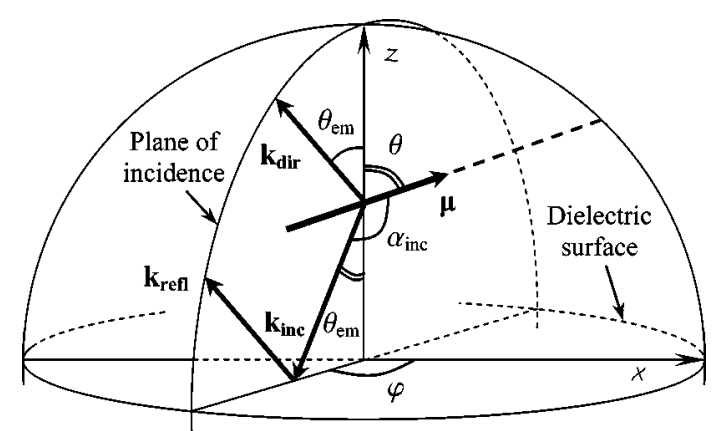

FIG. 2. Dipole emission model showing the direct, incident and reflected waves $\mathbf{k}_{\mathrm{dir}}, \mathbf{k}_{\mathrm{inc}}$ and $\mathbf{k}_{\mathrm{refl}}$. The emitting dipole is located in the $x-z$ plane.

where $k_{x}$ and $k_{y}$ are the propagation constants of the plane waves in the $x$ and $y$ directions, $k_{0}$ is the wave number of the topmost free-space layer $\left(=\omega \sqrt{\mu_{0} \varepsilon_{0}}\right)$. As a result, the total electric field in the topmost semi-infinite layer [Eq. (3)] can be written as the inverse Fourier transform of the sum of the Green's functions given in Eq. (1) and Eq. (2). This transformation can be interpreted, from a physical point of view, as the summing up of all the plane wave components, as well as other wave components such as surface waves, lateral waves, and leaky waves. Therefore, it is quite complex and cannot be performed analytically for most of the problems, if not all, except for a few trivial cases.

Having shown the general procedure to find the fields accurately anywhere in multilayer planar environment, we focus on finding the electric field expressions for the spectral self-interference microscopy where only far-field expressions are necessary. Then the above mentioned inverse transform integral is approximated analytically using the stationary phase method, resulting in the following direct, incident, and reflected waves for the TE wave components

$$
\begin{aligned}
& E_{\mathrm{TE} d i r}=E_{\mathrm{TE} i n c} \propto \sin \theta \sin \varphi, \\
& E_{\mathrm{TE} r e f l}=E_{\mathrm{TE} i n c} R_{\mathrm{TE}} e^{i 2 \phi},
\end{aligned}
$$

and for the TM wave components

$$
\begin{aligned}
& E_{\mathrm{TM} d i r} \propto \cos \theta_{e m} \sin \theta \cos \varphi-\sin \theta_{e m} \cos \theta, \\
& E_{\mathrm{TM} i n c} \propto \cos \theta_{e m} \sin \theta \cos \varphi+\sin \theta_{e m} \cos \theta, \\
& E_{\mathrm{TM} r e f l}=E_{\mathrm{TM} i n c} R_{\mathrm{TM}} e^{i 2 \phi},
\end{aligned}
$$

where $\phi=(2 \pi n / \lambda) d \cos \theta_{e m}, n$ is the refractive index of the medium where the dipole is located, $d$ is the distance of the dipole above a stack of dielectric layers, $\theta$ is the polar tilt of the dipole in the $x-z$ plane, $\theta_{e m}$ is the emission angle (or polar angle of the observation point), and $\varphi$ is the azimuthal angle of the observation point, all of which are shown in Fig. 2. In spectral self-interference microscopy, the spectral shape of the emission is important rather than the absolute emission intensity. Therefore only relative angular dependence of the fields are considered and the proportionality sign $(\propto)$ is used. Once all the field components are calculated, the total fields for the TE and TM waves at the observation point are simply found as

$$
E_{\mathrm{TE}}=E_{\mathrm{TE} d i r}+E_{\mathrm{TE} r e f l}
$$

$$
E_{\mathrm{TM}}=E_{\mathrm{TM} d i r}+E_{\mathrm{TM} r e f l},
$$

and then, the emission intensity of the dipole in the direction characterized by $\theta_{e m}$ and $\varphi$ is obtained:

$$
I=\left|E_{\mathrm{TE}}\right|^{2}+\left|E_{\mathrm{TM}}\right|^{2} \text {. }
$$

The total emission of a monolayer of random dipoles measured through an objective with maximum collection angle $\theta_{e m}^{\max }$ is calculated by integrating the emission intensity over the objective:

$$
I_{\text {total }}=\int_{\theta=0}^{\pi / 2} \int_{\varphi=0}^{\pi} \int_{\theta_{e m}=0}^{\theta_{e m}^{\max }} I\left(\theta, \varphi, \theta_{e m}\right) \sin \theta_{e m} d \theta_{e m} d \varphi d \theta .
$$

If there are additional dielectric layers between the dipole and the objective, that is, fluorophore is embedded in a planar cavity, then the emission of the dipole is modeled in two steps. First, the field propagating upward is calculated as if there were no layers above the fluorophore, by the sum $\mathbf{E}_{0}=\mathbf{E}_{\mathrm{dir}}+\mathbf{E}_{\mathrm{refl}}$, and then, the resulting field is multiplied by the total transmission coefficient that takes into account both the multiple reflections between the top and the bottom interfaces inside the cavity, and the transmission to the objective. Hence, the field right at the objective is obtained as

$$
E=E_{0} T^{\prime} /\left(1-R^{\prime} R^{i 2 \phi}\right), \quad \phi=\frac{4 \pi n}{\lambda} D \cos \theta_{e m},
$$

where $R^{\prime}$ and $T^{\prime}$ are the generalized reflection and transmission coefficients for the layers above the fluorophore in the direction towards the objective, $R$ is the generalized reflection coefficient of the layers below the fluorophore, and $D$ and $n$ are the thickness and the index of refraction of the cavity containing the fluorophore.

Once the model is developed, the interpretation of selfinterference spectra can be easily automated with an algorithm that splits the spectrum into its two major components: the envelope and the oscillatory curve. Only the parameters for the oscillatory curve are used as variables in the trial-anderror fitting process, and the spectral data is then divided by the computer-generated curve. If the parameters are correct, the resulting product should be an envelope function free of oscillations. The smoothness of the reconstructed envelope can be monitored by fitting it with a polynomial function and determining the least squares deviation. The point where the deviation goes through a minimum corresponds to the oscillatory curve parameters that are closest to actual ones. With the classical model of dipole emission described above, this algorithm usually provides an excellent fit to the experimental self-interference data (Fig. 3).

An exception to a good fit can be found for the case of a thick layer of fluorophores. The previous section has considered the emission from a single dipole, but the same description can be used for a distribution of dipoles, where the intensity from each dipole is added (incoherent sources). For a laterally extended dipole layer, there is no discernable difference from the single dipole, since the collection angles are not affected. However, an axially extended source region greatly affects the result. Adding the oscillating spectra from 


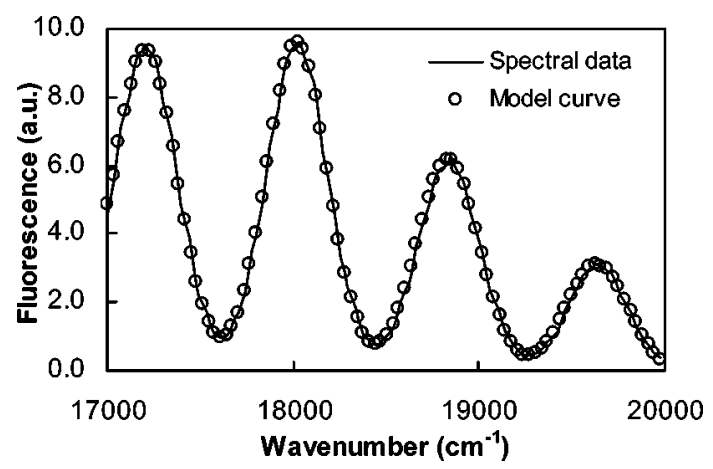

FIG. 3. Fitting of the experimental data. The reflectivity of the $\mathrm{Si}-\mathrm{SiO}_{2}$ surface was given by well-established indices of refraction. The only fitted variable was the thickness of the oxide layer. Fluorescein layer was located directly on top of the chip.

different axial locations results in a spectrum that reflects the intensity weighted average position of the fluorophore distribution. If the axial extent of the source region is several $10 \mathrm{~s}$ of nanometers, there will be a noticeable decrease in the contrast in the interference fringes. For fluorescent layers with thickness comparable to $\lambda$, interference curves will be washed out completely.

\section{EXPERIMENTAL RESULTS AND DISCUSSION}

To demonstrate the validity of the approach presented, spectral self-interference fluorescence microscopy was used to measure the position of a fluorescent monolayer above a silicon mirror. Three different types of fluorescent samples were prepared; fluorescein on top of $\mathrm{SiO}_{2} / \mathrm{Si}$ substrates, quantum dots (QDs) on top of $\mathrm{SiO}_{2} / \mathrm{Si}$ substrates, and a QD layer buried inside $\mathrm{SiO}_{2}$ on $\mathrm{SiO}_{2} / \mathrm{Si}$ substrates. Fluorescein isothiocyanate was immobilized on an aminosilane (APTES)-covered $\mathrm{Si}-\mathrm{SiO}_{2}$ chip with the thickness of the transparent $\mathrm{SiO}_{2}$ layer $\approx 4 \mu \mathrm{m}$. A monolayer of quantum dots was prepared on another chip by the technique described by Chan et al. ${ }^{9}$ Fluorescence emission spectra were acquired by a grating spectrometer equipped with a $5 \times$ objective $(N A=0.12)$ at 20 different positions on the chip. Fluorescent signal was collected from a spot 10-20 micrometers in diameter. The $\mathrm{SiO}_{2}$ thickness on the substrate is constant to within $0.3 \mathrm{~nm}$ across the $20 \mu \mathrm{m}$ sample spot. Even though the sample spot is relatively large $(10-20 \mu \mathrm{m})$, it is still very small compared to the diameter of the objective lens, and therefore does not affect the collection angles described in section III. Hence, only a single lateral point in the objective focus needs to be considered. The thickness of the oxide layer was determined by white light interference measurements at the same location on the chip. White light interference determines the thickness of a transparent film on top of a reflecting surface by measuring the complex reflectivity of the dielectric stack as a function of wavelength. ${ }^{5}$ White light reflectivity measurements yield the optical cavity thickness, $n D$, similar to the results of ellipsometry. The selfinterference fluorescence technique is different in that it is measuring the axial location of the fluorophore, regardless of how sparse the fluorophores are and whether they are buried inside a layer or on top of a surface. a)



b)

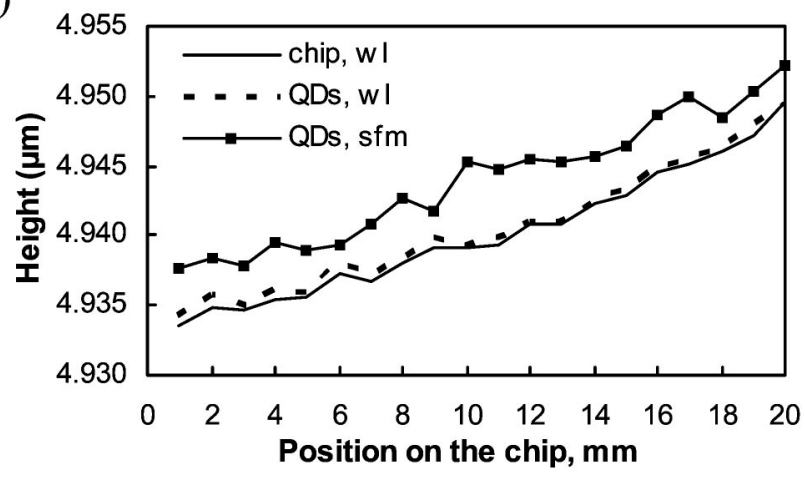

FIG. 4. (a) The average position of the fluorescent emitters above the surface of a $\mathrm{Si}_{-} \mathrm{SiO}_{2}$ chip. (b) White light and SFM measurements of a $\mathrm{Si}-\mathrm{SiO}_{2}$ chip before and after quantum dot deposition. Quantum dots are rare enough so that they almost do not change the optical thickness of the transparent layer on the Si mirror. SFM measurements show the source of emission is about $3 \mathrm{~nm}$ above the surface. The overall slope of the curve is due to the varying thickness of the underlying $\mathrm{SiO}_{2}$ layer across the area of the scan $(10 \mathrm{~mm})$.

The dipole orientation in all experiments was assumed to be isotropic in the claculations. Integrations over dipole orientation and polar and azimuthal angles of emitted waves were done by summing up the electrical field over the integration range with a $5^{\circ}-10^{\circ}$ step. This resulted in 10-20 integration slices for a $\pi / 2$ range for polar dipole orientation.

Figure 4(a) shows the schematics of the sample. If the layer of quantum dots is sparse enough, the effect of the quantum dots on determining the thickness of the transparent $\mathrm{SiO}_{2}$ layer is insignificant. White light measurements of the chip before and after quantum dot deposition show an addition of only a few Ångströms. Fluorescence interference measurements collected from the same position on the chip show the average height of the emitters above the surface is $3 \mathrm{~nm}$, about half the size of a quantum dot. On the other hand, if the emitter is a small molecule, such as fluorescein, the SFM measurements pinpoint its position to be within $1 \mathrm{~nm}$ from the surface of the chip (data not shown). Hence, the location of fluorophores can be determined relative to an interface to within a few angströms, even for a sparse layer of fluorophores.

The notable advantage of this technique is that the apparent position of emitters does not depend on the overall emission intensity. Also, the shape of the envelope has no effect on the fitting routine, as long as it does not contain oscillations similar to those produced by self-interference.

The model describes the emission of a flat monolayer of fluorescent dyes. If the sample contains a vertical distribution of fluorophores, it is necessary to integrate the signal from all layers. 
If the fluorophores are isolated and immobile, it is also necessary to consider the excitation strength as a function of dipole orientation. Although the above model is capable of incorporating the excitation strengths of individual dipoles, the total emission calculation in Eq. (11) assumes that all dipoles in the monolayer are equally excited, and therefore, equally contributing to the emission of the monolayer of dipoles.

It is essential to take into account the polarization effects associated with dipole orientation. If random dipoles are considered isotropic emitters for simplicity, the contrast of the interference oscillations deviates from the model by several percent.

Buried layers of quantum dots for subsurface measurements were prepared by covering the chip with an additional layer of silicon dioxide by electron beam deposition. The emission profile of quantum dots and their position inside a silicon-silicon oxide-air cavity was also correctly predicted by the classical model.

\section{CONCLUSION}

Spectral self-interference is an improved technique for measuring the height of fluorescent molecules above a re- flecting surface. A classical model of dipole emission that takes polarization effects into account can be used to precisely describe the oscillatory spectral component of fluorophores whether they are located outside or buried inside the transparent spacer layer of silicon oxide.

\section{ACKNOWLEDGMENTS}

This work was supported by NSF under Grant No. DBI 0138425 and by NIH under Grant No. 5R01 EB00 756-03.

${ }^{1}$ O. Wiener, Ann. Phys. (Leipzig) 40, 203 (1890).

${ }^{2}$ K. H. Drexhage, Prog. Opt. 12, 163 (1974).

${ }^{3}$ A. Lambacher and P. Fromherz, Appl. Phys. A: Mater. Sci. Process. 63, 207 (1996)

${ }^{4}$ J. Eschner, Ch. Raab, F. Schmidt-Kaler, and R. Blatt, Nature (London) 413, 495 (2001).

${ }^{5}$ A. K. Swan, L. Moiseev, C. R. Cantor, B. Davis, S. B. Ippolito, W. C. Karl, B. B. Goldberg, and M. S. Ünlü, JSTQE 9, 294 (2003).

${ }^{6}$ G. Dural and M. I. Aksun, IEEE Trans. Microwave Theory Tech. 43, 7 (1995).

${ }^{7}$ W. C. Chew, Waves and Fields in Inhomogeneous Media (Van Nostrand Reinhold, New York, 1990), Chap. 2.1, p. 49.

${ }^{8}$ Private communication with L. Novotny.

${ }^{9}$ W. C. W. Chan, D. J. Maxwell, X. H. Gao, R. E. Bailey, M. Y. Han, and S. M. Nie, Curr. Opin. Biotechnol. 13, 40 (2001). 